03

\title{
О квантовой интерпретации рассеяния света вблизи границы прозрачных сред с керровской нелинейностью
}

\author{
(C) А.В. Белинский \\ Московский государственный университет имени М.В. Ломоносова, физический факультет, \\ 119991 Москва, Россия \\ e-mail: belinsky@inbox.ru
}

Поступила в редакцию 10.06.2017 г.

В окончательной редакции 19.01.2018 г.

\begin{abstract}
Рассмотрено преобразование света нелинейным светоделителем. Он представляет собой плоскую границу раздела двух прозрачных диэлектриков, по крайней мере, один из которых обладает керровской нелинейностью, т. е. зависимостью показателя преломления от интенсивности пронизывающего его излучения. Показано, что интерпретация результата расчета квантовых полей на выходах такого светоделителя свидетельствует о нарушении принципа причинности в смысле влияния последующего события на предыдущее.
\end{abstract}

DOI: $10.21883 /$ OS.2018.05.45951.110-17

\section{Введение}

Понятие причинности имеет несколько значений. Первое из них ретроспективно: квантовая наблюдаемая, не находящаяся в собственном состоянии измеряемой величины, в серии экспериментов принимает различные значения, подчиняющиеся вероятностной статистике. Что предопределяет конкретное значение измеренной величины в единичной реализации? Эйнштейн, Подольский и Розен [1] считали, что это делает некая совокупность скрытых параметров, которыми следует дополнить квантовую теорию. Бор настаивал на принципиальной непредсказуемости результата [2] и полноте квантовой теории без всяких скрытых параметров. Эта точка зрения и получила свое экспериментальное подтверждение в серии работ, начиная с Белла [3-10]. В этом смысле можно говорить о „беспричинности“ результата квантовых измерений, поскольку принципиально невозможно его заранее предсказать [2].

Другое значение понятия причинности связано со световым конусом и специальной теорией относительности (СТО): следствие не может наступить раньше, чем причина, причем скорость причинно-следственной связи между ними не должна превышать световую. Однако целый ряд экспериментов с так называемым отложенным выбором и квантовым ластиком опровергли и это понятие причинности: скорость причинно-следственной связи в них на несколько порядков превышала световую $[11,12]$. Но следствие при этом не опережало причины.

Следующий по значимости эффект с точки зрения причинности связан с квантовым парадоксом Зенона $[13,14]$. В нем два последовательных квантовых перехода как бы нарушают причинность в самом широком смысле этого слова: второй по времени переход определяет вероятность первого. В самом деле, единичная вероятность второго перехода обращает в ноль вероятность первого, т. е. полностью блокирует его. Но с другой стороны, нулевая вероятность первого также блокирует второй, поэтому скорее следует говорить об их равноправности, поскольку вероятность обоих переходов является результатом интерференции амплитуд вероятностей обоих переходов, т.е. квадратом модуля суммы их комплексных амплитуд.

Рассмотренный далее эффект в определенном смысле переворачивает причину и следствие во времени, что ведет к нарушению причинности в самой радикальной форме. Он связан с квантовым парадоксом нелинейного светоделителя [15-20], в котором фазовые флуктуации света возникают как бы из ничего [21]. Позже появилась работа, развивающая прикладную направленность этого явления [22]. Однако дальнейшее исследование парадокса приводит к еще более интересным следствиям, которые уже касаются фундаментальных основ квантовой теории, и ее интерпретаций.

Чтобы легко войти в смысл задачи, рассмотрим нелинейный светоделитель, представляющий собой границу раздела двух прозрачных диэлектриков (рисунок). Пусть один из них обладает кубической керровской самофокусирующей нелинейностью, обусловливающей увеличение показателя преломления с ростом интенсивности излучения. Коэффициенты пропускания и отражения такой границы раздела изменяются с изменением интенсивности света в соответствии с формулами Френеля. Пусть линейная среда предшествует нелинейной, и показатель преломления второго диэлектрика в рабочем режиме, т. е. с учетом нелинейной добавки, превышает показатель преломления первого: тогда с увеличением интенсивности излучения увеличивается и показатель преломления, следовательно, увеличивается коэффициент отражения границы раздела, а коэффициент пропускания снижается. Следовательно, флуктуационное увеличение интенсивности входного пучка будет частично компенсироваться уменьшением коэффициента пропускания. Таким образом, происходит насыщение или некоторое „запирание“ системы, приводящее к стабилизации интенсивности 
прошедшего света. Ощутимая стабилизация может проявляться и в отраженном от границы раздела пучке, если показатель преломления с увеличением интенсивности излучения, напротив, уменьшается, либо изменено соотношение показателей преломления: первый больше второго.

Рассмотрим одномодовый случай плоских монохроматических волн. Фаза плоской волны вблизи плоской границы раздела прозрачных сред, отраженной либо прошедшей, в классическом описании инвариантна с точностью до возможного скачка на $\pi$ - таковой в отраженном пучке. При этом складывается интересная ситуация: амплитудные флуктуации прошедшего либо отраженного пучка могут уменьшаться, а фазовые должны оставаться неизменными, что, казалось бы, приведет к нарушению принципа неопределенности Гейзенберга. Однако квантовый расчет показывает, что это не так: фазовые флуктуации увеличиваются, хотя с классической точки зрения непонятно за счет чего [19-21]. Здесь мы исследуем результаты квантового подхода с точки зрения их интерпретации. Дело в том, что итоговый результат - число зарегистрированных фотонов на выходах светоделителя - зависит от значения нелинейной добавки к показателю преломления. Но до момента регистрации число фотонов не определено [14]. Таким образом, получается, что конкретное значение нелинейности появляется только после регистрации фотонов на выходах. Но детектирование происходит после прохождения светом светоделителя. Следовательно, нарушается принцип причинности в самом общем его виде, когда последующее событие определяет предыдущее. Такова краткая логика рассуждений, более подробно рассмотренная далее.

Чтобы не учитывать фазового набега в прозрачных средах, рассматриваем пучки именно вблизи (на расстоянии не более длины волны) границы раздела. Кроме того, выделение такого узкого слоя (например, в результате размещения его между двумя плоскопараллельными пластинами из линейных материалов) избавляет от эффекта неустойчивости плоской волны в среде с самофокусирующей нелинейностью, являющегося классическим результатом нелинейной оптики, поскольку на малой длине распространения света неустойчивости не возникает

\section{1. Классическое описание светоделителя}

Рассмотрение преломления света границей раздела двух сред естественно начать с закона Снеллиуса:

$$
n_{1} \sin \vartheta_{1}=n_{2} \sin \vartheta_{2},
$$

и формулы Френеля для амплитудного коэффициента отражения [23]:

$$
\rho=-\frac{\sin \left(\vartheta_{1}-\vartheta_{2}\right)}{\sin \left(\vartheta_{1}+\vartheta_{2}\right)}
$$

которая справедлива в случае плоско поляризованного света с вектором напряженности электрического поля, перпендикулярным плоскости чертежа (так называемая $s$-поляризация). Для другой, взаимно ортогональной поляризации ( $p$-поляризации), синусы заменяются тангенсами.

Если нет потерь, то коэффициенты пропускания и отражения связаны простым соотношением:

$$
\tau^{2}+\rho^{2}=1,
$$

следующим из закона сохранения энергии. Сразу отметим, что анализируются только случаи режимов прохождения света, а полное внутреннее отражение из рассмотрения исключается.

Нелинейность одной из граничащих сред означает зависимость ее свойств от интенсивности пронизывающего ее излучения. Например, керровская нелинейность влияет на показатель преломления. В случае первой нелинейной среды

$$
n_{1}=n_{10}+\chi(\sqrt{m}-\sqrt{l})^{2}, \quad n_{2}=\mathrm{const},
$$

поскольку в первой среде одновременно присутствуют и падающая, и отраженная волны. Они складываются когерентно, в отклике среды возникают интерференционные нелинейные члены, и в общем случае волны становятся кноидальными, однако последние возникают в объеме среды, а мы рассматриваем тонкий слой вблизи границы раздела, где фазовый набег отраженной волны либо отсутствует, либо равен $\pi$ - при отражении от более плотной среды. В последнем случае в формуле (1.4a) следует брать разность амплитуд $(\sqrt{m}-\sqrt{l})$, а при отражении от менее плотной среды - сумму $(\sqrt{m}+\sqrt{l})$.

Для второй нелинейной среды ситуация проще:

$$
n_{2}=n_{20}+\chi k, \quad n_{1}=\mathrm{const},
$$

где $n_{i 0}$ - показатель преломления в темноте, а $\chi-$ коэффициент, пропорциональный кубической нелинейности среды $\chi^{(3)}$. Для удобства сопоставления с квантовым подходом интенсивность света условно измеряем числами фотонов в некотором объеме квантования. Эти безразмерные величины, разумеется, пропорциональны интенсивностям рассматриваемых плоских монохроматических волн.

Совместное решение системы уравнений (1.1)-(1.4) с учетом очевидных соотношений для комплексных амплитуд прошедшей и отраженной волн:

$$
a_{i}=\tau a_{i}, \quad a_{r}=\rho a_{i},
$$

даст искомое соотношение между входной и выходными волнами. Индексы $t, r, i$ относятся к проходящему, отраженному и падающему пучкам.

Аналитического решения, к сожалению, получить не удается. Поэтому для исследования статистических характеристик такого пассивного нелинейного преобразователя приходится прибегать к численному эксперименту [21]. Но, как это ни странно, квантовое описание все же допускает возможность аналитического решения. 


\section{2. Квантовое решение в представлении Шрёдингера}

Пусть светоделитель (рисунок) освещается слева модой в фоковском состоянии $|m\rangle$ с определенным числом фотонов $m$. Но потенциально у него есть и другой вход - сверху. Даже при отсутствии на нем излучения там будет вакуум $|0\rangle$. Если бы светоделитель был линейным, то состояние на выходе описывалось бы вектором [24]:

$$
|\psi\rangle=\sum_{k=0}^{m} \sqrt{C_{k}^{m}} \tau^{k} \rho^{m-k}|k\rangle|l\rangle,
$$

- прошло $k$ фотонов, отразилось $l=m-k, \tau$ и $\rho-$ френелевы амплитудные коэффициенты пропускания и отражения, $C_{k}^{m}-$ биномиальный коэффициент.

Это соотношение является точным квантовым результатом, однако оно имеет простой физический смысл: фотоны в данном случае ведут себя как обычные классические не взаимодействующие частицы. Например, вероятность прохождения одного фотона равна $\tau^{2}$, а $k$ фотонов $-\tau^{2 k}$. Вероятность того, что прошло $k$ фотонов, а отразилось $l=m-k$ равна произведению $\left(\tau^{2}\right)^{k}\left(\rho^{2}\right)^{m-k}$. Биномиальный коэффициент $C_{k}^{m}=\frac{m !}{k !(m-k !)}$ появляется вследствие эквивалентности всех возможных сочетаний прошедших и отраженных фотонов. А поскольку вектор состояния представляет собой не саму вероятность, а ее амплитуду, извлекаем квадратный корень.

Нелинейность можно учесть функциональной зависимостью $\tau$ и $\rho$ от $m \pm l$ в случае первой нелинейной среды, а второй - линейной и от $k-$ в случае обратного их расположения. В случае двух граничащих между собой нелинейных сред обе зависимости будут присутствовать одновременно. При этом можно использовать обычные формулы для $\tau$ и $\rho(1.2)$ и $(1.3)$ с учетом

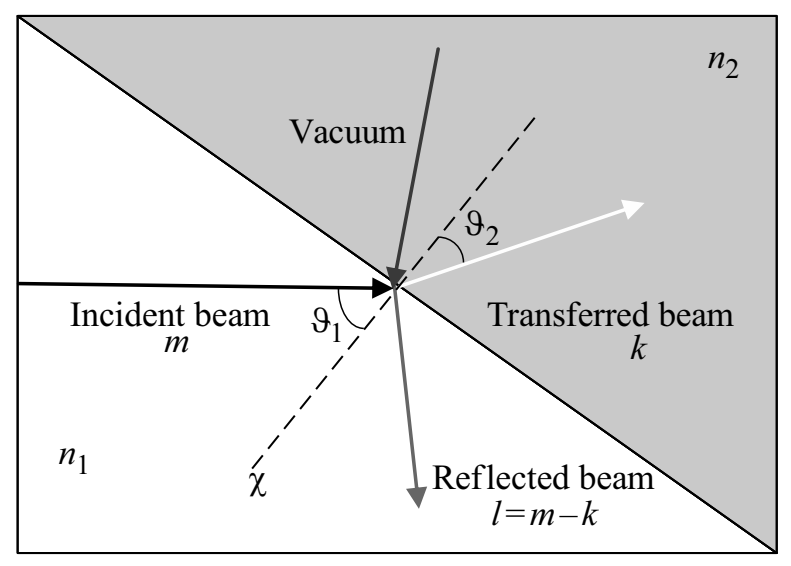

Нелинейный светоделитель со второй нелинейной средой, $\vartheta_{1}$ и $\vartheta_{2}-$ углы падения и преломления. На первом входе когерентная мода с плоским волновым фронтом, на втором вакуум. того, что нелинейная добавка показателя преломления пропорциональна числу фотонов в среде, а коэффициент пропорциональности $\chi$, в свою очередь, пропорционален коэффициенту кубической нелинейности $\chi^{(3)}$.

При произвольном состоянии на входе |\rangle , например когерентном $|z\rangle$, его можно разложить по фоковскому базису:

$$
|\rangle=\sum_{m=0}^{\infty} D_{m}|m\rangle
$$

тогда

$$
\begin{gathered}
|\psi\rangle \propto \sum_{m=0}^{\infty} D_{m} \sum_{k=0}^{m} \sqrt{C_{k}^{m}} \tau^{k}(m \pm l) \rho^{l}(m \pm l)|k\rangle|m-k\rangle \\
\equiv \sum_{m=0}^{\infty} \sum_{k=0}^{m} \Lambda_{k l}|k\rangle|l\rangle \\
|\psi\rangle \propto \sum_{m=0}^{\infty} D_{m} \sum_{k=0}^{m} \sqrt{C_{k}^{m}} \tau^{k}(k) \rho^{l}(k)|k\rangle|m-k\rangle \\
\equiv \sum_{m=0}^{\infty} \sum_{k=0}^{m} \Lambda_{k l}|k\rangle|l\rangle
\end{gathered}
$$

Здесь (a) и (b) отличаются аргументами величин и в зависимости от той или иной последовательности расположения нелинейной и линейной сред, о чем было сказано выше. В силу нелинейности задачи приходится производить перенормировку, чтобы выполнялось $\langle\psi \mid \psi\rangle=1$.

Физический смысл квадратов коэффициентов $\Lambda_{k l}^{2}=\mid\left\langle l|\langle k|| \psi\rangle|^{2}\right.$ очень прост: это вероятность того, что пройдет $k$ фотонов и одновременно отразится $l$, т.е. мы имеем дело с двумерной условной вероятностью. Теперь нетрудно найти распределения вероятностей обнаружить определенное число фотонов в отраженном и проходящем пучках:

$$
P_{l}=\sum_{k=0}^{\infty} \Lambda_{k l}^{2}, \quad P_{k}=\sum_{l=0}^{\infty} \Lambda_{k l}^{2} .
$$

В этом переходе от линейной задачи к нелинейной есть одна очень важная тонкость. Если на входе было фоковское состояние, то на выходах, разумеется, нет: число фотонов $k$ и $l$ может быть разным. Точнее, выходные состояния представляют собой суперпозицию фоковских. Так какому же из них должна соответствовать нелинейность? Будем рассуждать операционально. Если мы детектируем на выходах фотоны, то в каждой реализации получим вполне определенное их число $k$ и $l$. Именно этим числом и будет определяться нелинейность, что и отражено в (2.3). И на входе их было, таким образом, тоже определенное число $m=k+l$.

Хотя формально здесь все верно, возникает вопрос: откуда нелинейность светоделителя заранее „знает“, сколько фотонов будет зарегистрировано на выходах? 
Ведь если поле внутри светоделителя находится в состоянии суперпозиции фоковских состояний, то, значит, в нем присутствуют все фоковские моды [14,25]. Так какие из них должна „выбрать“ нелинейность, чтобы сошлись концы с концами? Без опережающего знания о последующем детектировании выходных полей это просто невозможно. Значит, нарушается принцип причинности в том смысле, что последующее событие определяет предыдущее, что вполне укладывается в рамки реляционно-статистической природы закономерностей квантовой теории [26].

\section{Заключение}

Предложен оценочный квантовый расчет преобразования бозонных полей нелинейным светоделителем, представляющим собой плоскую границу раздела двух прозрачных сред, одна из которых (или обе) обладает кубической керровской нелинейностью, т.е. показатель преломления зависит от интенсивности пронизывающего излучения. Вообще говоря, последовательный вывод потребовал бы квантового описания в рамках макроскопического подхода, включая описание оптического возбуждения - фотона/поляритона - в материальной среде. Ввиду нековариантной природы макроскопического усреднения строго это выполнить невозможно, но если принять справедливость приведенных оценок, то получается следующее.

Характерным свойством квантовых состояний, не являющихся собственными состояниями измеряемой величины, оказывается наличие квантовой суперпозиции всех возможных результатов измерений. Если рассуждать операционально, то для описания такой нелинейнооптической квантовой задачи нужно начинать с конца - с количества фотонов, зарегистрированных на выходах светоделителя. Затем, „раскручивая“ процесс в обратном (по отношению к реально текущему времени) направлению, определить число фотонов в нелинейнооптической среде светоделителя и соответствующую нелинейность. После чего задача получает аналитическое решение. Но, как часто бывает в квантовых задачах, возникает вполне закономерный с точки зрения „здравого смысла“ вопрос: что происходит между рождением квантовой частицы и ее регистрацией? Что первично: нелинейность, формирующая выходные поля светоделителя, или количество фотоосчетов, которые определяют нелинейность? Если следовать хронологическому порядку, то ясно, что первое. Но до момента регистрации (априори) определенного числа фотонов в нелинейной среде не существует, а есть лишь квантовая суперпозиция фоковских состояний. Значит, и определенной нелинейности не существует до момента финальной регистрации частиц. Итак, последующее по времени событие определяет предыдущее. А это несомненно нарушает принцип причинности в самой общей его формулировке.
С другой стороны, в современной квантовой теории поля, подтверждаемой множеством экспериментов, эта проблема давно является частью конкретных расчетов. Во внутренних диаграммных разложениях инвариантной теории возмущений всегда присутствует причинная функция Грина как пропагатор любой виртуальной квантовой частицы. Как простой наглядный пример, можно отметить формулу Крамерса-Гейзенберга для рассеяния фотона на атоме, где вполне допускается появление рассеянного фотона раньше исчезновения рассеиваемого фотона. При этом нет необходимости интерпретировать промежуточные виртуальные состояния как реально существующие физические объекты.

Автор благодарен М.Х. Шульману за сотрудничество и помощь в работе. Работа поддержана грантом РФФИ № 18-01-00598.

\section{Список литературы}

[1] Einstein A., Podolsky B., Rosen N. // Phys. Rev. 1935 V. 47. P. 777.

[2] Bohr N. The Causality Problem in Atomic Physics // In: New Theories in Physics. Paris, 1939. Перевод: УФН. 1985. Т. 147. № 2. C. 343.

[3] Bell J.S. // Physics. 1964. V. 1. P. 195.

[4] Aspect A., Grangier P., Roger G. // Phys. Rev. Lett. 1981. V. 47. P. 460.

[5] Aspect A., Grangier P., Roger G. // Phys. Rev. Lett. 1982 V. 49. P. 91.

[6] Aspect A., Dalibar J., Roger G. // Phys. Rev. Lett. 1982 V. 49. P. 1804.

[7] Hensen B., Bernien H., Dre'au A.E. et al. // Nature. 2015. doi 10.1038/nature15759

[8] Giustina M., Versteegh M.A.M., Wengerowsky S. et al. // Phys. Rev. Lett. 2015. V. 115. P. 250401.

[9] Groblacher S., Paterek T., Kaltenbaek R. et al. // Nature. 2007. V. 446. P. 871.

[10] Белинский А.В. // Вестник Московского университета. Cер. 3. Физика, астрономия. 2016. № 5. С. 26.

[11] Ma X., Kofler J., Zeilinger A. // Rev. Mod. Phys. 2016. V. 88. P. 015005.

[12] Ma X., Kofler J., Qarry A. et al. // Proc. Natl. Acad. Sci. USA. 2013. V. 110. P. 1221.

[13] Менский М.Б. Квантовые измерения и декогеренция. М.: Физматлит, 2001. 232 с.

[14] Белинский А.В. Квантовые измерения. М.: БИНОМ: Лаборатория знаний, 2008. $182 \mathrm{c.}$

[15] Белинский А.В. // Письма в ЖЭТФ. 1990. Т. 51. № 7. C. 341.

[16] Белинский А.В. // Квант. электрон. 1991. Т. 18. С. 84.

[17] Белинский А.В., Грановский А.А. // Письма в ЖЭТФ. 2011. T. 93. C. 552.

[18] Белинский А.В., Волков Д.В., Дмитриев А.В., Шульман М.X. // ЖЭТФ. 2013. Т. 144. С. 891.

[19] Фобанов Я. А. // Опт. и спектр. 2003. Т. 94. № 5 С. 861; Fofanov Ya.A. // Opt. Spectrosc. 2003. V. 94. P. 802. 
[20] Fofanov Ya.A. // ICONO 2010: International Conference on Coherent and Nonlinear Optics. / Ed. by Claude F., Zadkov A., Drabovich V.K. // Proc. SPIE. 2011. V. 7993. P. 79930.

[21] Белинский А.В., Шульман М.Х. // УФН. 2014. Т. 184. C. 1135.

[22] Prakash H., Kumar Mishra D. // JOSA. B. 2016. V. 33. P. 1552.

[23] Борн М., Вольф Э. Основы оптики. М.: Наука, 1970. 720 с.

[24] Leonhardt U. Measuring the Quantum State of Light, Cambridge University Press, 1997. 204 p.

[25] Белинский А.В., Жуковский А.К. // Вестник Московского университета. Сер.3. Физика, астрономия. 2016. № 3. С. 34.

[26] Белинский А.В., Владимиров Ю.С. // Пространство, время и фундаментальные взаимодействия. 2016. № 1(14). С. 32. 